\title{
An Idea about Negative Cosmic Time in the Big Bang-Big Rip Cosmological Model
}

\author{
M. A. Bakry ${ }^{1, *}$, A. Eid ${ }^{2,3,+}$ and A. Alkaoud ${ }^{2, * *}$ \\ ${ }^{1}$ Department of Mathematics, Faculty of Education, Ain Shams University, Cairo, Egypt \\ ${ }^{2}$ Department of Physics, College of Science, Imam Mohammad Ibn Saud Islami University (IMSIU), \\ Riyadh, KSA \\ ${ }^{3}$ Department of Astronomy, Faculty of Science, Cairo University, Giza, Egypt
}

\begin{abstract}
In this article, we assume that the beginning of the universe was before the Big Bang. In the beginning, all matter in the universe was combined in an infinitesimal spherical shape. This sphere was compressed to an incomprehensible value for a period, and then exploded and expanded time and space. We are referring to the negative time before the Big Bang. The evolution of the universe before the Big Bang, passing through the moment of the explosion to the end of the universe at the Big Rip, has been studied. In this article, we try to answer the questions; did the universe exist before the Big Bang? What is the origin of the universe and how did it arise? What are the stages of the evolution of the universe until the moment of the Big Rip? What is the length of time for the stages of this development?

Keywords: Negative cosmic time; cosmological solutions; variable deceleration parameter; big rip.
\end{abstract}

PACS Nos. : 04.20.- q, 11.10.-z, 98.80.Es, 98.80.-k.

*mohamedbakry928@yahoo.com

+amaid@imamu.edu.sa

**akaoud@imamu.edu.sa 


\section{Introduction}

Based on the fact that the universe began with the Big Bang, many researchers have used multiple universe models to study the evolution of the universe [1-6]. The aim of this article is to study the behavior of the universe before and after the moment of a Big Bang. We denote negative time as the period before the "Big Bang", and positive time as the period after the explosion. The study relies on cosmic parameters to understand the physical behavior of the evolution of the universe and compare the results with the available observational information. It has been proclaimed in 1998, that type of observations of Ia supernovae indicate that the current universe is not only expanding but also accelerating. This was maybe the most arresting discovery of modern cosmology. Now, this behavior of the universe has been confirmed by various independent observational data, including Type Ia supernova (SNIa), large structures (SDSS), and the cosmic microwave background (CMB) radiation, and so on. In a recent paper, we will review the compression of the universe's matter until the moment of the Big Bang, and then the stages of expansion to the Big Rip of the universe. In order to crystallize the organization of the current manuscript, the rest of the paper is organized as follows: Section $\mathbf{2}$ is devoted to investigating the Physical evolution of the universe. Comparison with the observed kinematics of the universe is illustrated in Section 3. The discussions of the results are provided in Section 4. Finally, the concluding remarks are drawn in Section $\mathbf{5}$.

The Einstein field equations can be written as follows [7],

$$
G_{\alpha \beta}=R_{\alpha \beta}-\frac{1}{2} g_{\alpha \beta} R=T_{\alpha \beta},
$$

where $G_{\alpha \beta}$ is the Einstein tensor and $T_{\alpha \beta}$ is the energy-momentum tensor. Considering a comoving fluid the perfect fluid representation for the energy-momentum tensor, this can be written as follows,

$$
T_{\beta}^{\alpha}=[\rho, p, p, p],
$$

where $\rho$ is the energy density of the matter in co-moving coordinates and $p$ is pressure.

In order to solve the Einstein field equations, it is usually necessary to make some simplifying assumptions such as selecting a metric with a important degree of symmetry. One may be considered the Robertson-Walker $(R W)$ metric with a maximally symmetric spatial section [8],

$$
d s^{2}=d t^{2}-S^{2}(t)\left(\frac{d r^{2}}{W(r)}+r^{2}\left(d \theta^{2}+\sin ^{2} \theta d \phi^{2}\right)\right),
$$

where $W(r)=1-K r^{2}, S(t)$ is the cosmic scale factor, and the spatial curvature index $K \in\{-1,0,1\}$ corresponds to spatially open, flat and closed universes, respectively.

The Einstein field equations (1) with (2) and (3), leads to [9],

$$
\begin{aligned}
\rho & =\frac{3 \dot{S}^{2}+3 K}{S^{2}}, \\
p & =-\left(\frac{\dot{S}^{2}+K+2 S \ddot{S}}{S^{2}}\right),
\end{aligned}
$$

Most of the perfect fluids relevant to cosmology obey an equation of state of the form $p=\omega \rho$, thus the state parameter is,

$$
\omega=-\left(\frac{\dot{S}^{2}+K+2 S \ddot{S}}{3 \dot{S}^{2}+3 K}\right) .
$$


The three most common examples of cosmological fluids with constant $\omega$ are the dust $(\omega=0)$, radiation $(\omega=1 / 3)$ and vacuum energy $(\omega=-1)$. It is well known that fluids with $(\omega<-1 / 3)$ are usually considered in the context of dark energy $(D E)$, since they give rise to accelerating expansion.

Akarsu and Dereli have been proposed the generalized, linearly varying deceleration parameter, as follows [1],

$$
q=\frac{d H^{-1}}{d t}-1=m-1-a t
$$

Where $a \geq 0$ and $m \geq 0$ are constants.

They proposed the deceleration parameter as a function of the first degree in time to find a finite cosmological model that ends at the moment of the Big Rip.

Now, the Hubble parameter of the universe is obtained as follows:

$$
H=\frac{\dot{S}}{S}=\frac{2}{t(2 m-a t)} .
$$

Equation (8), gives

$$
\begin{aligned}
& \dot{S}=\frac{2 S}{t(2 m-a t)}, \\
& \ddot{S}=\frac{4 S(1-m+a t)}{t^{2}(2 m-a t)^{2}} .
\end{aligned}
$$

Accordingly, integrating Eq. (8), one obtains [6],

$$
S(t)=S_{0}\left(\frac{t}{2 m-a t}\right)^{1 / m} \text {. }
$$

Substituting from Eqs.(9), (10), and (11) into (4),(5), and(6), one gets

$$
\begin{aligned}
& \rho=\frac{12 t^{2 / m}+3 K t^{2}(2 m-a t)^{2+\frac{2}{m}}}{(2 m-a t)^{2} t^{2+\frac{2}{m}}}, \\
& p=-\left(\frac{4 t^{2 / m}(3-2 m+2 a t)+K t^{2}(2 m-a t)^{2+\frac{2}{m}}}{(2 m-a t)^{2} t^{2+\frac{2}{m}}}\right),
\end{aligned}
$$

equation of state parameter $(E O S) \omega=p / \rho$ can be obtained in a straight for-ward manner from Eqs. (12), and (13) as

$$
\omega=-\left(\frac{4 t^{2 / m}(3-2 m+2 a t)+K t^{2}(2 m-a t)^{2+\frac{2}{m}}}{12 t^{2 / m}+3 K t^{2}(2 m-a t)^{2+\frac{2}{m}}}\right) .
$$

One can be also solve for the deceleration parameter $q$ as a function of the redshift $z+1=S_{z=0} / S, S_{z=0}$ is the present value of the scale factor

$$
q(z)=m-1-\frac{2 m a S_{z=0}^{m}}{\left((1+z)^{m}+a S_{z=0}^{m}\right)},
$$

where $S_{z=0}=2.455$, see table 1 . 
It is believed that the transition from the decelerating to the accelerating phase of the Universe comes due to a Cosmic Jerk as defined by the Jerk parameter. This parameter, in the Cosmology, is defined as $[10,11]$,

$$
j(t)=\frac{1}{S H^{3}} \frac{d^{3} S}{d t^{3}}=q(t)+2 q^{2}(t)-\frac{1}{H(t)} \frac{d q(t)}{d t} .
$$

One may obtain the Jerk parameter $J(t)$, as follows

$$
j(t)=1+(a t-m)(3+2 a t-2 m)-a t(a t-2 m) / 2 .
$$

\section{Physical evolution of the universe}

In this section, we will study the physical behavior of the evolution of the Universe. Big Bang cosmology is a $R W$ solution based on general relativity. This is a very successful model; it provides a reliable, tested history of the universe, about $10^{=2}$ seconds after the explosion, until today, some $15 \mathrm{Gyr}$ later. It is so successful that it is known as the standard model of cosmology. It accommodates-and in some instances explains-most of the salient features of the observed universe, including the Hubble expansion, the $2.74 \mathrm{~K}$ cosmic microwave background radiation (CMBR), the abundance of the light elements, and the existence of structures like galaxies, clusters of galaxies, etc. So now we are able to ask a new set of even more profound questions about the universe. Did the universe exist before the Big Bang? What are the essence of the universe and the nature of pressure? What is his evolutionary behavior when he reached that "Big Bang" moment? And so on. To answer these questions, we will take a model that represents the evolution of the universe from the moment of the Big Bang to the moment of the Big Rip and take it back using the negative time "before the beginning of time at the Big Bang" and study the evolutionary behavior of the universe during a long period similar to the theoretically estimated age of the universe.

In our model the universe has finite lifetime. The universe began with its deceleration parameter to $q=3 m-1$, and $S(t)=\left(\frac{-1}{2 a}\right)^{1 / m}$ at $t=-\frac{2 m}{a}$, and then gradually reach to $q=m\left(1+\frac{a}{3}\right)-1$, and $S(t)=\left(\frac{-1}{6+a}\right)^{1 / m}$ at $t=-\frac{m}{3}$ and enters to Big Bang with $q=m-1$, and $S(t)=0$ at $t_{b b}=0$, this is the Big Bang behavior, then moved to acceleration when $q=0$, and $S(t)=\left(\frac{m-1}{m+1}\right)^{1 / m}$ at $t_{a}=\frac{m-1}{a}$, then to strong acceleration with $q=-1$, and $S(t)=\left(\frac{1}{a}\right)^{1 / m}$ at $t_{s a}=\frac{m}{a}$, the universe ends at the moment of a Big Rip with deceleration parameter $q=-(m+1)$, and $S(t)=\infty$ at $t_{b r}=\frac{2 m}{a}$. This is the big rip behavior first suggested by [1, and 12]. From equation (15), the relationship between the redshift and the deceleration parameter can be found as follows

$$
z=S_{z=0}\left(a\left(\frac{2 m}{m-1-q}-1\right)\right)^{1 / m}-1,
$$

In the next section, we will display the previous introductions numerically so that we can compare them with the observed observations. 


\section{Comparison with the observed kinematics of the universe}

In order to demonstrate how our model matches the observed kinematics of the universe and makes additional predictions, we first plot the cosmological parameters by choosing $m=2$ and $a=0.126$, comparison with reference [1,13, and 14]. From Eq.(11) as well as the plot of scale factor $S(t)$ (see Fig. 1), It is easy to observe that at the beginning (i.e. $t=-31.8$ ) i.e. universe start evolving with volume $|S(t)|=1.99$, then it looked and continued to shrink until it reached zero volume $S(t)=0$ with an infinite rate of expansion at $t=0$ or at Big Bang. The universe continued to expand until it reached its maximum value at the end (i.e. $t=31.8$ or at Big-Rip). Figure 2 depicts the Hubble parameter $H(t)$ for our mode. It is clear that the Hubble parameter is beginning small value at $t=-31.8$, and then it reaches its highest value at ( $t=0$ or at BigBang, also at $t=31.8$ or at Big-Rip).

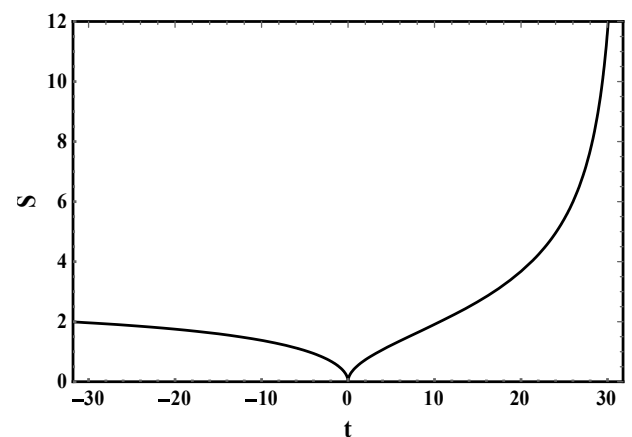

Figure 1: Scale factor $S(t)$ versus cosmic time $t:-31.8 \rightarrow 31.8$

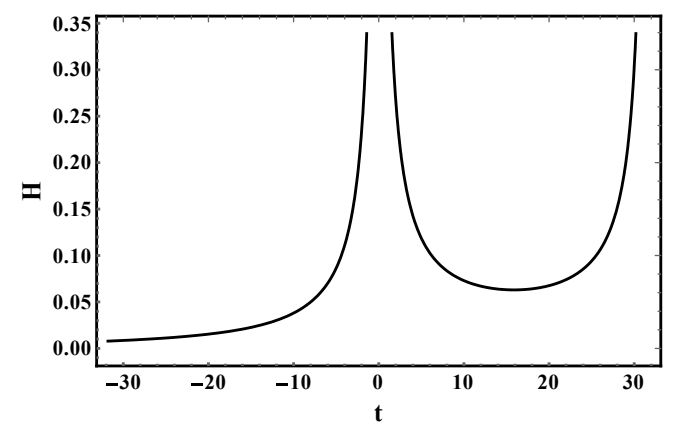

Figure 2: Hubble parameter $H$ versus cosmic time $t:-31.8 \rightarrow 31.8$

The deceleration parameter $q$ is positive (decelerating expansion) at early universe $(-31.8 \leq t \leq 7.9)$ and negative (accelerating expansion) at present time (see Fig. 3). It means universe indicate transitional phase (i.e. early time deceleration to late time acceleration). The universe began with its deceleration parameter to $q=5$, at $t=-31.8$, and then gradually reach to $q=1.13$, and at $t=-1$ and enters to Big Bang with $q=1$, at $t_{b b}=0$, this is the Big Bang behavior, then moved to acceleration when $q=0$, at $t_{a}=7.9$, and then enters to $q_{d a y}=-0.73$ at $t_{d a y}=13.7$, and then to strong acceleration with $q=-1$, at $t_{s a}=15.87$, the universe ends at the moment of a big rip with deceleration parameter $q=-3$, at $t_{b r}=31.8$.

We also plot the deceleration parameter $q(z)$ versus redshift $z$ in Fig. 4.From model dependent or independent analyses of the cosmological observations, the transition redshift of the accelerating expansion is given by $0.3<z_{t}<0.8[13,14,15-20]$. One may observe that the accelerating expansion in our model begins at $z \approx 0.51$ consistent with the observational data, for more details, see Refs. [21-28]. One cannot find a numerical value or observations of the redshift before and during the Big Bang. 


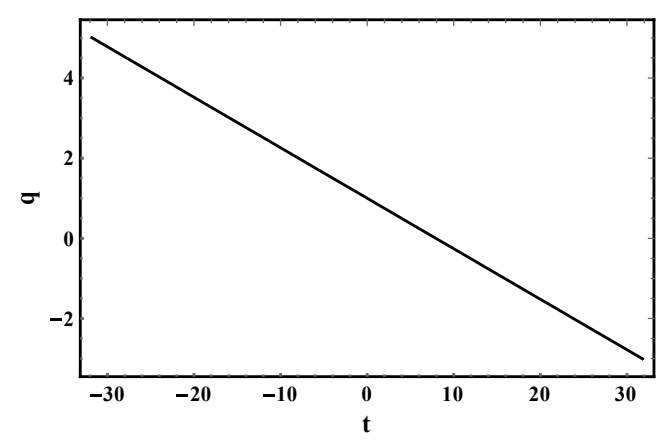

Figure 3: Deceleration parameter $q(t)$ versus cosmic time $t:-31.8 \rightarrow 31.8$

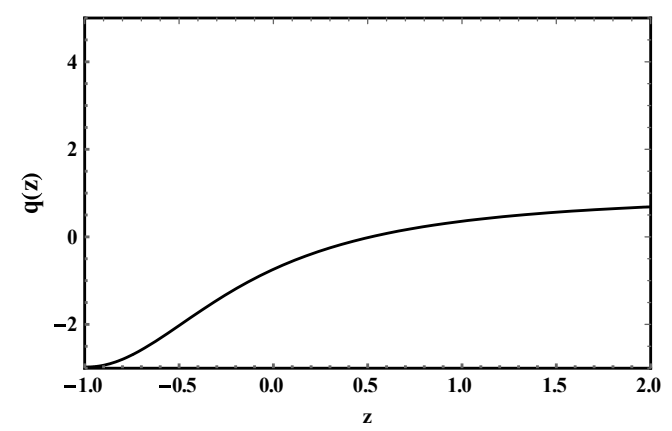

Figure 4: Deceleration parameter $q(z)$ versus redshift $z:-1 \rightarrow 2$

We plotted the energy density versus time $\rho(t)$ in Fig. 5 for our model. Concern figures indicate the flat model began with its energy density $\rho=1.9 \times 10^{-4}$, at $t=-31.8$, and then gradually reach to $\rho=0.71$ at $t=-1$ and enters to Big Bang with $\rho_{b b} \rightarrow \infty$ at $t_{b b}=0$, this is the Big Bang behavior, then moved to acceleration when $\rho_{a}=2.1 \times 10^{-2}$ at $t_{a}=7.9$, and then enters to $\rho_{\text {day }}=1.2 \times 10^{-2}$ at $t_{\text {day }}=13.7$, and then to strong acceleration with $\rho_{s a}=1.1 \times 10^{-2}$, at $t_{s a}=15.87$, the universe ends at the moment of a big rip with energy density $\rho_{b r} \rightarrow \infty$, at $t_{b r}=31.8$. One can accept this cosmological model because it satisfies the condition of positive energy density $\rho \geq 0$. In Fig. 6 we plot the pressure of the fluid $p$ versus cosmic time $t$. The universe began with positive pressure before the Big Bang. This pressure diverges at the "Big Bang" and the "Big Rip". The our flat model began with its pressure $p=5.6 \times 10^{-4}$, at $t=-31.8$, and then gradually reach to $p=0.29$ at $t=-1$ and enters to Big Bang with $p_{b b} \rightarrow \infty$, at $t_{b b}=0$, this is the Big Bang behavior, then moved to acceleration when $p_{a}=-7 X 10^{-3}$, at $t_{a}=7.9$, and then enters to $p_{d a y}=-1 . X 10^{-2}$ at $t_{d a y}=13.7$, and then to strong acceleration with $p_{s a}=-1.2 \times 10^{-2}$, at $t_{s a}=15.87$, the universe ends at the moment of a big rip with energy density $\rho_{b r} \rightarrow-\infty$, at $t_{b r}=31.8$.

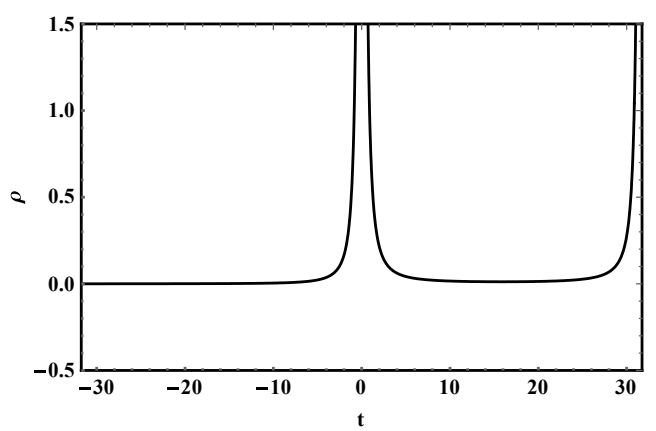

Figure 5: The energy density of the fluid $\rho$ versus cosmic time $t:-31.8 \rightarrow 31.8$, and $K=0$

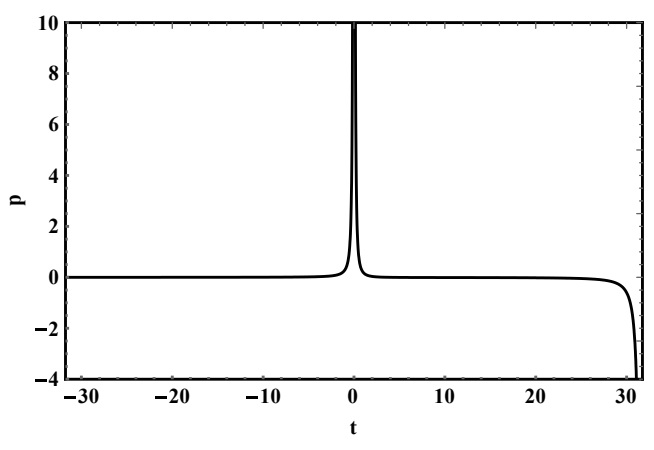

Figure 6: The pressure of the fluid $p$ versus cosmic time $t:-31.8 \rightarrow 31.8$, and $K=0$

In Fig. 7, we have plotted the evolution of $E o S$ parameter $\omega$, as a function of cosmic time. The flat model began with its state parameter $\omega=2.5$, at $t=-31.8$, and then gradually reach to $\omega=0.41$, and at $t=-1$ and enters to Big Bang with $\omega=1 / 3$, at $t_{b b}=0$, this is the Big Bang 
behavior, then moved to acceleration when $\omega=-1 / 3$, at $t_{a}=7.9$, and then enters to $\omega_{d a y}=-0.82$ at $t_{d a y}=13.7$, and then to strong acceleration with $\omega=-1$, at $t_{s a}=15.87$, the universe ends at the moment of a big rip with state parameter $\omega=-2.3$, at $t_{b r}=31.8$. Our model is consistent with the observed data of SNIa [29], Golden Sample of Hubble Space Telescope [30], CMB [31], and SDSS [32] because it allows the dark energy $(D E)$ to pass into the phantom region $(\omega<-1)$.In Fig. 8, we have plotted the jerk parameter with time $j(t)$, it is clear from the concern figure that the values of the jerk parameter correspond to the evolution of the universe and its transition from a state of deceleration to a state of acceleration.

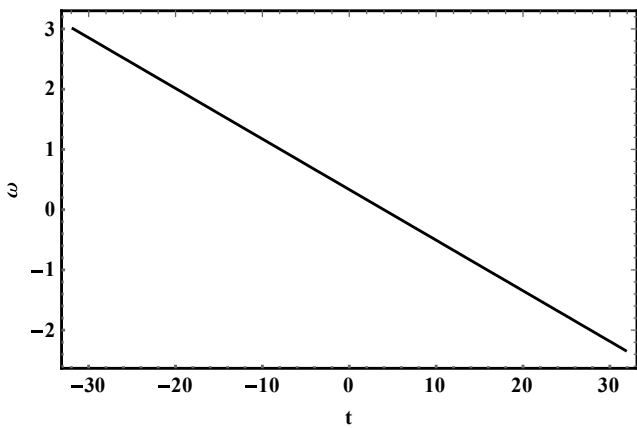

Figure 7: The equation of state parameter $\omega$ versus cosmic time $t:-31.8 \rightarrow 31.8$, and $K:-1 \rightarrow 1$

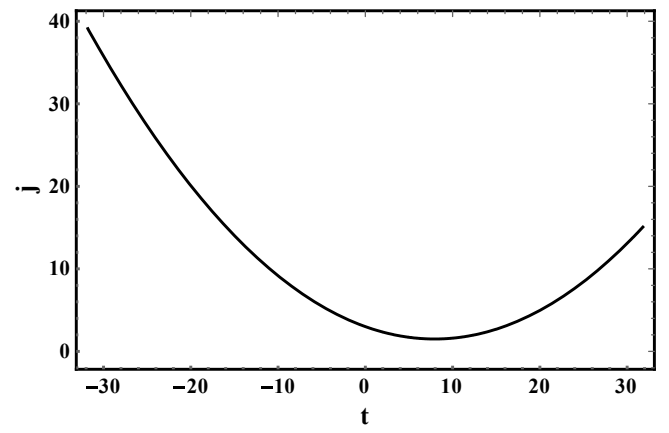

Figure 8: Jerk parameter $j(t)$ versus cosmic time $t:-31.8 \rightarrow 31.8$

\section{Discussions and results}

The aim of this article is to identify the behavior of the evolution of the universe before a moment of a Big Bang, so in section 1, we chose a linearly varying deceleration parameter model. We reviewed in this section, the cosmic scale factor, the deceleration parameter, the energy density of the matter, the pressure in the fluid, and the state parameter for this model. In section 2, we presented the Physical evolution of the universe from the supposed beginning of the universe, through the moment of the Big Bang, to the Big Rip. The results of this article can be presented in Table 1.

Table 1: Evolution of the universe in the proposed model

\begin{tabular}{|c|c|c|c|c|c|c|c|}
\hline The stages & $t$ & $q$ & $S$ & $p$ & $\rho$ & $\omega$ & $z$ \\
\hline \multirow[t]{6}{*}{ Before the Big Bang } & -31.8 & 5.00 & 1.99 & $5.6 \times 10^{-4}$ & $1.9 \times 10^{-4}$ & 2.50 & \\
\hline & -15.87 & 2.90 & 1.60 & $2.2 \times 10^{-3}$ & $1.3 \times 10^{-3}$ & 1.69 & \\
\hline & -13.70 & 2.71 & 1.55 & $2.9 \times 10^{-3}$ & $1.9 \times 10^{-3}$ & 1.53 & \\
\hline & -7.936 & 1.99 & 1.26 & $7.6 \times 10^{-3}$ & $7.6 \times 10^{-3}$ & 1.00 & \\
\hline & -3.968 & 1.50 & 0.92 & $2.5 \times 10^{-2}$ & $3.8 \times 10^{-2}$ & 0.66 & \\
\hline & -1.00 & 1.13 & 0.49 & 0.29 & 0.71 & 0.41 & \\
\hline Big Bang moment & 0.00 & 1.00 & 0 & $\rightarrow \infty$ & $\rightarrow \infty$ & $1 / 3$ & \\
\hline \multirow[t]{3}{*}{ After the Big Bang } & 1.00 & 0.87 & 0.51 & 0.199 & 0.799 & $1 / 4$ & \\
\hline & 3.968 & 0.50 & 1.29 & $\rightarrow 0$ & $6.2 \times 10^{-2}$ & 0 & \\
\hline & 7.936 & 0.00 & 1.62 & $-7 \times 10^{-3}$ & $2.1 \times 10^{-2}$ & $-1 / 3$ & 0.51 \\
\hline
\end{tabular}




\begin{tabular}{|c|c|c|c|c|c|c|c|}
\hline & 13.70 & -0.73 & 2.46 & $-1 \times 10^{-2}$ & $1.2 \times 10^{-2}$ & -0.82 & $-5.8 \times 10^{-3}$ \\
\cline { 2 - 7 } & 15.87 & -1.00 & 2.82 & $-1.2 \times 10^{-2}$ & $1.1 \times 10^{-2}$ & -1 & -0.13 \\
\hline Big Rip moment & 31.80 & -3.00 & $\rightarrow \infty$ & $\rightarrow-\infty$ & $\rightarrow \infty$ & -2.30 & -1 \\
\hline
\end{tabular}

In this article, we use the negative time to refer to just before the Big Bang. Before the Big Bang, it can be assumed that the size of the early universe contained pure water. This volume is under the influence of pressure that increases with the passage of time with a high temperature and high energy density. Under endothermic reactions the volume shrinks with water and decomposes into the elements $\mathrm{H}, \mathrm{O}, \mathrm{O}_{2}, \mathrm{OH}$, and $\mathrm{H}_{2}$. As the temperature and pressure increase, the preceding elements decompose into elementary particles with high energy. The pressure value increases with increasing temperature, and vice versa (This idea needs more scrutiny in other research that includes specialists in chemistry and physics), see Refs. [33 - 36]. According to the chemical and physical studies of thermal reactions during this period, the suggested time before the Big Bang can be shortened. A Big Bang occurs when both pressure and temperature reach their maximum value. One can note that the universe turns from a radiation state to a dust one in a period of about time $3.968 \mathrm{Gyr}$, and it begins to accelerate after 7.936 Gyr, from the moment of the Big Bang. From Table 1, one can be observed that the accelerating expansion begins at $z=0.51$, this result is consistent with the observational data. Also, the age of the universe is now estimated at about $13.70 \mathrm{Gyr}$. In our model, the phantom region starts at $t>15.87 \mathrm{Gyr}$. These results agree with the context of $D E$ in general relativity, see Refs.[37-40].

\section{Concluding remarks}

In this article, we propose a model of the universe that began before the Big Bang, and we call this period negative time. We have studied the behavior of the universe from the Big Bang to the Big Rip. The results of the proposed model are consistent with the results of astronomical observations so far. Many researchers have studied the early-time behavior of the Universe and in particular the Big Bang Nucleosynthesis (BBN) epoch, see Refs,[41-44]. But before the Big Bang, it needs an in-depth study by physicists and chemists under the conditions of the present study. In this article, we assumed that the origin of the universe was water, which decayed under the influence of pressure and high temperature into elementary elements that interacted with each other, and then the Big Bang occurred. Based on this, a Physico-chemical study is needed that determines the stages of the transformation of water into its elements, the time required for that transformation, and the interactions that occur with high pressure and temperature to very high levels. Our study of the universe means that the universe has an origin that it originated from and that it is a non-periodic universe.

Author Contributions: Conceptualization, M. A. Bakry, and A. Eid; investigation: M. A. Bakry, A. Eid, and A. Alkaoud; Methodology, M. A. Bakry, A. Eid; formal analysis, M. A. Bakry, and A. Eid; writing, M. A. Bakry, and A. Eid ; validation, M. A. Bakry, A. Eid, and A. Alkaoud, All authors have read and agreed to the published version of the manuscript.

Funding: This research was funded by Imam Mohammad Ibn Saud Islamic University, KSA, Research Group no. RG-21-09-42. 
Acknowledgment: The authors extend their appreciation to the Deanship of Scientific Research at Imam Mohammad Ibn Saud Islamic University, KSA for funding this work through Research Group no. RG-21-09-42. Also, the authors would like to express their deep gratitude to Prof. M. I. Wanas and Prof. M. I. Kahil for their deep interest and valuable comments during extraction of this work. The authors would like to express their deep gratitude to Dr. M for English language review.

Conflicts of Interest: The authors declare no conflict of interest

\section{References}

1. Akarsu, Ö.; Dereli, T. Cosmological models with linearly varying deceleration parameter. Int. J. Theo. Phys.2012, 51(2), 612.

2. A karsu, Ö.; Dereli, T. A Comparison of the LVDP and $\Lambda$ CDM Cosmological Models. Int. J.Theo. Phys.2012, 51(10), 2995.

3. Sahoo, P. K.; Sivakumar, M. LRS Bianchi type-I cosmological model in $f(R, T)$ theory of gravity with $\Lambda(\mathrm{T})$. Astrophys, Space Sci.2015, 357(1), 1.

4. Sahoo, P. K.; Tripathy, S. K.; Sahoo, P. A periodic varying deceleration parameter in $\mathrm{f}(\mathrm{R}, \mathrm{T})$ gravity. Mod. Phys. Lett. A 2018, 33, 1850193.

5. Chand, A.; Mishra, R. K.; Pradhan, A. FRW cosmological models in Brans-Dicke theory of gravity with variable $\$ \$$ and dynamical $\Lambda$ \$varLambda $\$$-term. Astrophys. Space Sci. 2016, 361(2), 81 .

6. Bakry, M. A.; Shafeek, A. T. The periodic universe with varying deceleration parameter of the second degree. Astrophys. Space Sci. 2019, 364(8), 1-6.

7. Weinberg, S. Gravitation and cosmology john wiley \& sons. Inc., USA.1972.

8. Robertson, H. P. Groups of motions in spaces admitting absolute parallelism. Ann. Math. 1932, 496.

9. Berman, M. S. Introduction to General Relativity and the Cosmological Constant Problem. Nova Publishers.2007.

10. Chiba, T.; Nakamura, T. The luminosity distance, the equation of state, and the geometry of the universe. Prog. Theo. Phys.1998, 100(5), 1077-1082.

11. Visser, M. Jerk, snap and the cosmological equation of state. Class. Quan. Grav.2004, 21(11), 2603.

12. Caldwell, R. R.; Kamionkowski, M.; Weinberg, N. N. Phantom energy: dark energy with $\mathrm{w}<-1$ causes a cosmic doomsday. Phys. Rev. Lett.2003, 91(7), 071301.

13. Cunha, J. V.; Lima, J. A. S. Transition redshift: new kinematic constraints from supernovae. Month. Not. Roy. Astrono. Soci.2008, 390(1), 210.

14. Cunha, J. V. Kinematic constraints to the transition redshift from supernovae type Ia union data. Phys. Rev. D 2009, 79(4), 047301.

15. Li, Z.; Wu, P.; Yu, H. Examining the cosmic acceleration with the latest Union2 supernova data. Phys. Lett. B 2011, 695(1-4), 1.

16. Frieman, J. A.; Turner, M. S.; Huterer, D. Dark energy and the accelerating universe. Annu. Rev. Astron. Astrophys. 2008, 46, 385.

17. Melchiorri, A.; Pagano, L.; Pandolfi, S. When did cosmic acceleration start?. Phys. Rev. D 2007, 76(4), 041301.

18. Ishida, E. E.; Reis, R. R.; Toribio, A. V.; Waga, I. When did cosmic acceleration start? How fast was the transition?. Astropar. Phys.2008, 28(6), 547.

19. Pandolfi, S. When did cosmic acceleration start?. Nuclear Physics B-Proceedings Supplements 2009, 194, 294. 
20. Lima, J. A. S.; Holanda, R. F. L.; Cunha, J. V. Are galaxy clusters suggesting an accelerating universe?. In AIP Conference Proceedings, 2010, 1241(1), 224). American Institute of Physics. 21. Virey, J. M.; Taxil, P.; Tilquin, A.; Ealet, A.; Tao, C.; Fouchez, D. Determination of the deceleration parameter from supernovae data. Phys. Rev. D 2005, 72(6), 061302.

22. Shapiro, C.; Turner, M. S. What do we really know about cosmic acceleration?. The Astrophys. J.2006, 649(2), 563.

23. Gong, Y.; Wang, A. Observational constraints on the acceleration of the Universe. Phys. Rev. D 2006, 73(8), 083506.

24. Astier, P. Can luminosity distance measurements probe the equation of state of dark energy?. Phys. Lett. B 2001, 500(1-2), 8.

25. Huterer, D.; Turner, M. S. Probing dark energy: Methods and strategies. Phys.Rev. D 2001, 64(12), 123527.

26. Weller, J.; Albrecht, A. Future supernovae observations as a probe of dark energy. Phys. Rev. D 2002, 65(10), 103512.

27. Alam, U.; Sahni, V.; Deep Saini, T.; Starobinsky, A. A. Is there supernova evidence for dark energy metamorphosis?. Mon. Not. Roy. Astron. Soc. 2004, 354(1), 275.

28. Nesseris, S.; Perivolaropoulos, L. Comparison of the legacy and gold type Ia supernovae dataset constraints on dark energy models. Phys. Rev. D 2005, 72(12), 123519.

29. Riess, A. G.; et al. Type Ia supernova discoveries at $\mathrm{z}>1$ from the Hubble Space Telescope: Evidence for past deceleration and constraints on dark energy evolution. The Astrophys. J. 2004, 607(2), 665 .

30. Astier, P.; et al. The Supernova Legacy Survey: measurement of, and w from the first year data set. Astro. Astrophys. 2006, 447(1), 31-48.

31. Komatsu, E.; et al. Five-year wilkinson microwave anisotropy probe observations: cosmological interpretation. The Astrophys. J. Suppl. Ser.2009, 180(2), 330.

32. Eisenstein, D. J.; et al. Detection of the baryon acoustic peak in the large-scale correlation function of SDSS luminous red galaxies. The Astrophys. J. 2005, 633(2), 560.

33. Funk, J. E. Thermochemical hydrogen production: past and present. Int. j. hyd. Ene.2001, 26(3), 185.

34. Ozbilen, A.; Dincer, I.; Rosen, M. A. Life cycle assessment of hydrogen production via thermochemical water splitting using multi-step $\mathrm{Cu}-\mathrm{Cl}$ cycles. J. Clea. Produ. 2012, 33, 202.

35. Feili, M.; Rostamzadeh, H.; Parikhani, T.; Ghaebi, H. Hydrogen extraction from a new integrated trigeneration system working with zeotropic mixture, using waste heat of a marine diesel engine. Int. J. Hyd. Ene.2020, 45(41), 21969.

36. Plaza, M.; Turner, C. Pressurized hot water extraction of bioactives. $\operatorname{Tr} A C$ Trends in Analytical Chemistry, 2015, 71, 39.

37. Carroll, S. M.; Hoffman, M.; Trodden, M. Can the dark energy equation-of-state parameter w be less than- 1?. Phys. Rev. D 2003, 68(2), 023509.

38. Copeland E. J.; Sami, M.; Tsujikawa, S. Dynamics of dark energy. J. Mod. Phys.D 2006, 15, 1753.

39. Zhao, G. B.; Xia, J. Q.; Li, H.; Tao, C.; Virey, J. M.; Zhu, Z. H.; Zhang, X. Probing for dynamics of dark energy and curvature of universe with latest cosmological observations. Phys. Lett. B, 2007, 648(1), 8.

40. Nesseris, S.; Perivolaropoulos, L. Crossing the phantom divide: theoretical implications and observational status. J. Cosm. Astropar. Phys., 2007, (01), 018.

41. Jedamzik, K. Big bang nucleosynthesis constraints on hadronically and electromagnetically decaying relic neutral particles. Phys. Rev. D 2006, 74(10), 103509. 
42. Kawasaki, M.; Kohri, K.; Moroi, T.; Yotsuyanagi, A. Big-bang nucleosynthesis and gravitinos. Phys. Rev. D, 2008, 78(6), 065011.

43. Arbey, A.; Auffinger, J.; Hickerson, K. P.; Jenssen, E. S. Alter BBN v2: A public code for calculating Big-Bang nucleosynthesis constraints in alternative cosmologies. Comp. Phys. Comm., 2020, 248, 106982.

44. Barrow, J. D.; Basilakos, S.; Saridakis, E. N. Big bang nucleosynthesis constraints on Barrow entropy. Phys. Lett. B, 2021, 815, 136134. 